\title{
Possible misidentification of Bacteroides sp., probably B. ureolyticus as Taylorella equigenitalis: Implications for the laboratory diagnosis of CEM
}

\author{
John E. MoOrE ${ }^{a *}$, B. Cherie MiLlaR ${ }^{\mathrm{a}}, \mathrm{Jiru}_{\mathrm{XU}}^{\mathrm{a}}$, \\ Thomas C. BUCKLEY ${ }^{b}$ \\ ${ }^{a}$ Northern Ireland Public Health Laboratory, Department of Bacteriology, Belfast City Hospital, \\ Belfast BT9 7AD, Northern Ireland \\ ${ }^{\mathrm{b}}$ Irish Equine Centre, Johnstown, Naas, Co. Kildare, Ireland
}

(Received 9 February 2001; accepted 21 June 2001)

\begin{abstract}
A wild-type isolate with similar morphological and phenotypic properties to Taylorella equigenitalis, the causative bacterial agent of contagious equine metritis (CEM), was referred for molecular identification by PCR amplification of the 16S rRNA gene. A species-specific PCR failed to yield a product compatible with that of $T$. equigenitalis. The direct sequencing of the universal $16 \mathrm{~S}$ rRNA PCR amplicon suggested the presence of a Bacteroides sp., probably Bacteroides ureolyticus, with no consequent effects on the movement and transportation of the animal. Adoption of such a molecular means of identification through sequencing may aid in the identification of the atypical forms of Taylorella equigenitalis, as recently described, as well as differentiating this species from Taylorella asinigenitalis.
\end{abstract}

horse / CEM / PCR / Taylorella equigenitalis / Bacteroides

Résumé - Possibilité d'erreur dans l'identification d'un Bacteroides sp., probablement B. ureolyticus, détecté comme Taylorella equigenitallis : conséquences pour le diagnostic en laboratoire de la métrite contagieuse équine. Un isolat de type sauvage ayant des caractéristiques morphologiques et phénotypiques similaires à celles de Taylorella equigenitallis, l'agent bactérien responsable de la métrite contagieuse équine, a été soumis pour identification moléculaire par la technique d'amplification en chaîne par la polymérase (PCR) du gène de l'ARNr 16S. Une PCR spécifique à l'espèce n'a pas permis d'obtenir un produit compatible avec celui de T. equigenitalis. Le séquençage direct de l'amplicon de PCR de l'ARNr 16 S universel suggère la présence d'un Bacteroides sp., probablement Bacteroides ureolyticus, n'ayant aucune conséquence sur le mouvement et le transport de l'animal. L'adoption d'une telle technique moléculaire d'identification par le séquençage pourrait aider à l'identification de formes atypiques de Taylorella equigenitalis qui ont été récemment décrites, ainsi que pour différencier cette espèce de Taylorella asinigenitalis.

cheval / amplification en chaîne par polymérase / métrite contagieuse équine / Bacteroides

* Correspondence and reprints

Tel.: (44) 289026 3554; fax: (44) 282589 2887; e-mail: jemoore @ niphl.dnet.co.uk 


\section{INTRODUCTION}

Contagious equine metritis (CEM) is a sexually transmitted disease of horses caused by the bacterium, Taylorella equigenitalis. Originally CEM was first reported in England in 1977 [4]. Since then, CEM and its causative agent have been detected in many countries and in various breeds of horses. CEM continues to be a cause for concern in veterinary microbiology, especially due to its economic implications. Diagnosis of CEM has been mainly based on the isolation of $T$. equigenitalis by bacteriological culture, with the central clitoral sinus in mares and the urethral fossa in stallions being the most likely locations, where this organism may be found. Usually this organism is cultured from swabs, by means of conventional selective bacteriological techniques. Growth requires between two to six days and is often difficult to observe due to competition from other commensal organisms in the equine genital tract.

We report a laboratory case of potential misidentification of Bacteroides sp., probably $B$. ureolyticus, as $T$. equigenitalis during routine screening of horses for CEM.

\section{MATERIALS AND METHODS}

\subsection{Bacteriological culture}

Routine swabs taken from the genital area of an animal were cultured on T. equigenitalis selective agar. For the conventional detection of $T$. equigenitalis, swabs were plated onto different media namely (i) CEMO agar (MAST DM470, MAST Diagnostics Ltd, Merseyside, England) containing streptomycin $(250 \mu \mathrm{g} / \mathrm{mL})$ and fungizone (5 $\mu \mathrm{g} / \mathrm{mL}$ ) (ii) CEMO agar supplemented with $5 \%, \mathrm{v} / \mathrm{v}$, chocolate and fungizone $(5 \mu \mathrm{g} / \mathrm{mL})$ and (iii) Columbia agar base supplemented with $5 \%$, v/v, defibrinated horse blood. All plates were incubated for 4-6 days $\left(37^{\circ} \mathrm{C}\right.$; $5 \%, \mathrm{v} / \mathrm{v}, \mathrm{CO}_{2}$ ).
A bacteriological culture, yielding a few small convex colonies, which grew on CEMO Selective Agar, with atypical colonial characteristics to Taylorella equigenitalis, i.e. greyish yellow, glistening with an entire edge, approximately $2-2.5 \mathrm{~mm}$ in diameter, but which shared several phenotypic characteristics, including Gram stain and morphology (Gram -ve rods), oxidase (+ve), microaerophilic growth (+ve in 5\%, $\mathrm{v} / \mathrm{v}, \mathrm{CO}_{2}$ ) and aerobic growth (-ve), was referred for molecular identification, as possibly being that of $T$. equigenitalis.

\subsection{DNA extraction}

Genomic bacterial DNA from the culture was obtained by employing the High Purity PCR template DNA extraction Kit (Roche Ltd., England) in accordance with the manufacturer's instructions.

\subsection{Ribosomal PCR amplification}

All reaction mixes were set up in a PCR hood in a room separate from that used to extract DNA and the amplification and postPCR room in order to minimise contamination. Reaction mixes ( $50 \mu \mathrm{L})$ were set up as follows: $-10 \mathrm{mM}$ Tris-HCl, $\mathrm{pH} 8.3,50 \mathrm{mM}$ $\mathrm{KCl}, 2.5 \mathrm{mM} \mathrm{MgCl}_{2}, 200 \mu \mathrm{M}$ (each) dATP, dCTP, dGTP and dTTP; $1.25 \mathrm{U}$ of Taq DNA polymerase (Amplitaq; Perkin Elmer), $0.2 \mu \mathrm{M}$ (each) of the universal 16S rRNA primers [12], i.e. forward primer: P11P, 5' GAG GAA GGT GGG GAT GAC GT 3' and reverse primer: P13P 5' AGG CCC GGG AAC GTA TTC AC 3' and $4 \mu \mathrm{L}$ of DNA template, containing approximately 50 ng DNA/mL DNA. After a "hot start", the reaction mixtures were subjected to the following thermal cycling sequence in a Perkin Elmer 2400 thermocycler: $96^{\circ} \mathrm{C}$ for 3 min followed by 40 cycles of $96^{\circ} \mathrm{C}$ for $1 \mathrm{~min}, 55^{\circ} \mathrm{C}$ for $1 \mathrm{~min}, 72{ }^{\circ} \mathrm{C}$ for $1 \mathrm{~min}$, followed by a final extension at $72{ }^{\circ} \mathrm{C}$ for 10 min. During each run, molecular grade 
water was included randomly as negative controls and appropriate DNA templates from $T$. equigenatilis were included as positive controls when appropriate.

\subsection{PCR confirmation by specific PCR}

Genomic DNA was amplified by means of a species-specific PCR for T. equigenitalis, as previously described [2]. A positive control of $T$. equigenitalis and a negative control of molecular grade water (Biowhittaker Inc., USA) were included as appropriate controls.

\subsection{Detection of PCR products}

Following amplification, aliquots $(15 \mu \mathrm{L})$ were removed from each reaction mixture and examined by electrophoresis $(80 \mathrm{~V}$, $45 \mathrm{~min}$ ) in gels composed of $2 \%(\mathrm{w} / \mathrm{v})$ agarose (Gibco, UK) in TAE buffer ( $40 \mathrm{mM}$ Tris, $20 \mathrm{mM}$ acetic acid, $1 \mathrm{mM}$ EDTA, $\mathrm{pH}$ 8.3), stained with ethidium bromide $(5 \mu \mathrm{g} / 100 \mathrm{~mL})$. Gels were visualised under UV illumination by means of a gel image analysis system (UVP Products, England) and all images archived as digital graphic files (*.bmp).

\subsection{Sequencing of amplicons and analysis of sequence data}

Amplicons chosen for sequencing were purified by means of a QIAquick PCR purification kit (Qiagen Ltd., UK) eluted in Tris- $\mathrm{HCl}$ (10 mM, pH 8.5) before sequencing, particularly to remove dNTPS, polymerases, salts and primers. The forward universal primer [5' GAG GAA GGT GGG GAT GAC GT 3'] was used for sequencing with the ABI PRISM ${ }^{\mathrm{TM}}$ Dye Terminator Cycle Sequencing Reaction with AmpliTaq DNA Polymerase ${ }^{\circledR}$, FS (PE Biosystems, Foster City, CA, USA) $\left(96{ }^{\circ} \mathrm{C}\right.$ for $1 \mathrm{~min}$, followed by 25 cycles of $96^{\circ} \mathrm{C}$ for $10 \mathrm{~s}$,
$50{ }^{\circ} \mathrm{C}$ for $5 \mathrm{~s}, 60{ }^{\circ} \mathrm{C}$ for $4 \mathrm{~min}$, then held at $4{ }^{\circ} \mathrm{C}$ ). The products were ethanol-precipitated and analysed on an ABI 373 Automatic Sequencer (PE Biosystems, Foster City, CA, USA). Campylobacter jejuni NCTC11168 and T. equigenitalis were included as positive sequencing controls. The resulting sequences obtained were compared with those stored in the Genbank Data system by means of BLAST alignment software [1] (http://www.blast.genome.ad.jp/).

\section{RESULTS}

The species-specific PCR failed to yield a product compatible with $T$. equigenitalis (Fig. 1). The isolate produced an amplicon of the correct size with the universal $16 \mathrm{~S}$ rRNA primers P11P/P13P (Fig. 2) and the sequencing of the amplified 216bp PCR amplicon derived from the query isolate suggested the presence of a Bacteroides sp., possibly B. ureolyticus.

\section{DISCUSSION}

This study describes a laboratory case of uncertainty over the identification of an organism growing on CEMO selective agar under microaerophilic conditions. Although the colonial characteristics of the colonies did not closely resemble $T$. equigenitalis and hence were atypical in this respect, they shared several other phenotypic properties with $T$. equigenitalis. As atypical variants of $T$. equigenitalis have been recently described in mares [8] and taking into consideration the economic importance of CEM, it was decided to delay transportation of the animal from where the swabs originated pending a detailed molecular work-up of the identification of the suspected colonies.

The genus Taylorella was first proposed in 1983 by Sugimoto et al. [10]. It was the strain first isolated and described by Taylor et al. [11] and accepted by the UK 


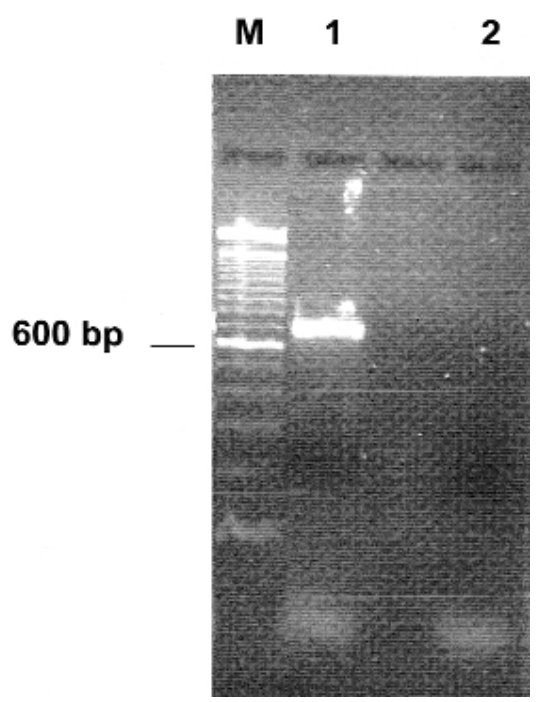

Figure 1. Species-specific PCR for Taylorella equigenitalis. Lane M: molecular weight marker (100 bp ladder; Gibco Ltd., Scotland, UK). Lane 1: positive control Taylorella equigenitalis. Lane 2: query isolate.

National Collection of Type Cultures (NCTC), as the Type Strain NCTC 11184 which was originally included as a species incertae sedis in the genus Haemophilus in Bergey's Manual of Determinative Bacteriology [7].

Phenotypically, the species shares characteristics of organisms in Group 4B (Gramnegative aerobic/microaerophilic rods and cocci) within Major Category I (Gram-negative Eubacteria that have cell walls) according to the 9th Edition of Bergey's Manual of Determinative Bacteriology, along with species in the genera Wolinella and Bacteroides. Phenotypically, species in the genera Taylorella and Bacteroides are very similar, being non-motile, microaerophilic and oxidase positive (B. ureolyticus), as described (Tab. I), although the latter genus has little clinical significance in CEM, whereas Taylorella is highly significant in this disease state. In addition, although phenotypically similar, T. equigenitalis is

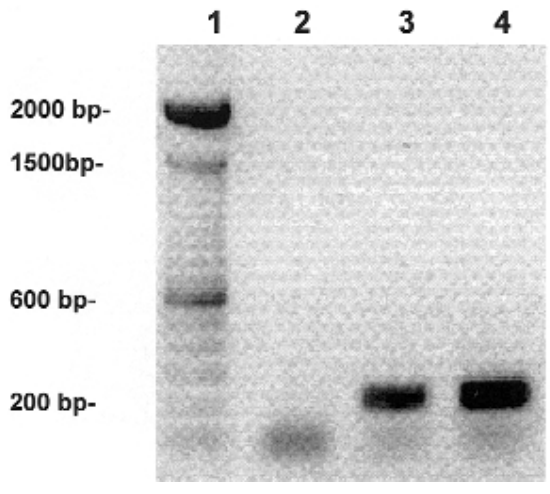

Figure 2. Gel electrophoresis of PCR products which resulted following amplification of a $216 \mathrm{bp}$ region of the $16 \mathrm{~S}$ rRNA gene from prepared genomic DNA. Lane 1: 100-bp DNA molecular weight ladder (Gibco, UK). Lane 2: negative control (molecular grade water). Lane 3: positive control (Taylorella equigenitalis). Lane 4: query isolate.

markedly different phylogenetically from Bacteroides ureolyticus. Analysis of the $16 \mathrm{~S}$ rRNA nucleotide sequence of $T$. equigenitalis (Genbank Accession No. X68545) and B. ureolyticus (Genbank Accession No. L04321) using alignment software (DNASTAR Inc., Wisconsin, USA) showed $63.7 \%$ homology between these two organisms.

Initially, it was surprising to detect a Bacteroides sp. on CEMO agar grown microaerophically, as species within this genus are regarded as being obligate anaerobes. This is largely true for the genus with the exception of $B$. ureolyticus, which is microaerophilic and hence could grow in $5 \% \mathrm{CO}_{2}$ conditions, which are normally achieved in common $\mathrm{CO}_{2}$ laboratory incubators. Indeed, this species of Bacteroides will not grow in anaerobic conditions. Furthermore this organism has been previously cultured from vaginal discharges from mares on three previous occasions $[3,5,6]$. In the 
Table I. Differential characteristics of Taylorella equigenitalis and Bacteroides ureolyticus.

\begin{tabular}{lcc}
\hline Phenotypic characteristic & Taylorella equigenitalis & Bacteroides ureolyticus \\
\hline Bergey's Classification & Major category I & Major category I \\
& Subgroup 4B & Subgroup 4B \\
Morphology & Rods & Rods \\
Gram reaction & Gram-negative & Gram-negative \\
Motility & Non-motile & Non-motile \\
Temperature growth range & $30-42^{\circ} \mathrm{C}$ & $25-37^{\circ} \mathrm{C}$ \\
Oxygen requirements & microaerophilic & microaerophilic \\
Oxidase & positive & positive \\
Carbohydrates utilised & none & none \\
Natural habitat & Genital tract & Urogenital tract \\
\hline
\end{tabular}

Fodor et al. study [5], seven cultures resembling $T$. equigenitalis were grown in microaerophilic conditions, which were confirmed as B. ureolyticus. All isolates were catalase positive, oxidase positive and alkaline phosphatase positive, being similar to T. equigenitalis. This study concluded that the Bacteroides ureolyticus organisms isolated closely resembled $T$. equigenitalis. Overall, this study demonstrated that both species are very similar in terms of their phenotypic characteristics, however differences included urease production by $B$. ureolyticus, leucine arylamidase and alanine arylamidase production by T. equigenitalis. For those laboratories which do not have access to molecular techniques, these differential phenotypic characteristics may be a useful aid in helping with laboratory identification.

The isolation of T. equigenitalis may prove difficult since the species is fastidious, requiring nutritionally-supplemented media and specialised reduced oxygen conditions. Furthermore, it is easily outgrown by other non-pathogenic organisms in the microflora found including commensal organisms in the genital tract of horses and hence may be misidentified in routine practice.

We report here on a case of possible misidentification of Bacteroides sp., as T. equigenitalis.
Direct sequencing of the 16S rRNA locus from the query isolate allows to identify a Bacteroides sp., possibly B. ureolyticus. As the PCR amplicon was 216 bp, only a relatively short region of the $16 \mathrm{~S}$ rRNA locus was amplified and sequenced. Hence it was not possible to differentiate the sequence identification lower than the genus level, without the need to sequence a larger stretch of this gene. However, the subsequent BLAST search led to the identification of Bacteroides sp.

Adoption of such a molecular means of identification through sequencing may aid in the identification of the atypical forms of Taylorella equigenitalis, as recently described [8], as well as differentiating this species from Taylorella asinigenitalis (GenBank Accession number AF067729 ) [9].

The correct identification of $T$. equigenitalis as the aetiological agent responsible for CEM is very important, as CEM requires special control measures and restrictions have to be introduced to prevent the transmission of disease locally, nationally and internationally, whereas colonisation/infection of the genital tract with Bacteroides spp. do not necessitate such measures.

With the adoption of highly discriminatory molecular methods such as PCR and direct automated sequencing into routine clinical microbiology, in cases of difficulty in identifying atypical colonies, we 
therefore recommend molecular identification by species-specific PCR amplification of a locus specific for T. equigenitalis [2], as well as by sequencing the $16 \mathrm{~S}$ rRNA locus after attempts to identify suspect colonies with a combination of biochemical (e.g. urease production, API Identification System) and serological testing have proved unsuccessful.

\section{REFERENCES}

[1] Altschuk S.F., Madden T.L., Schäffer A.A., Zhang J., Zhang Z., Miller W., Lipman D.J., Gapped BLAST and PSI-BLAST: a new generation of protein database search programs, Nucleic Acids Res. 25 (1997) 3389-3402.

[2] Anzai T., Eguchi M., Sekizaki T., Kamada M., Yamamoto K., Okuda, T., Development of a PCR test for rapid diagnosis of contagious equine metritis, J. Vet. Med. Sci. 61 (1999) 1287-1292.

[3] Buckley T.C., Leadon, D.P., A survey of anaerobic bacteria isolated from the cervico-endometrial swabs taken from mares present in Ireland during the 1986 thoroughbred breeding season, Ir. Vet. J. 42 (1988) 28-31.

[4] Crowhurst R.C., Genital infection in mares, Vet. Rec. 100 (1977) 476.

[5] Fodor L., Szenci O., Peters J., Varga J., Szemerédi G.Y., Wyszoczky F., Isolation of Bacteroides ureolyticus from vaginal discharge of mares, J. Vet. Med. B. 42 (1995) 415-420.
[6] Hariharan H., Richardson G., Horney B., Heaney S., Bryenton J., Moore I., Isolation of Bacteroides ureolyticus from the equine endometrium, J. Vet. Diagn. Invest. 6 (1994) 127-130.

[7] Holt J.G., Krieg N.R., Sneath P.H.A., Staley J.T., Williams S.T., Gram-negative aerobic/ microaerophilic rods and cocci, in: Bergey's manual of determinative bacteriology, 9th ed. Williams \& Wilkins, London, 1994, pp. 102.

[8] Katz J.B., Evans L.E., Hutto D.L., SchroederTucker L.C., Carew A.M., Donahue J.M., Hirsh D.C., Clinical, bacteriologic, serologic, and pathologic features of infections with atypical Taylorella equigenitalis in mares, J. Am. Vet. Med. Assoc. 216 (2000) 1945-1948.

[9] Moore J.E., Millar B.C., Buckley T.C., Possible misidentification of Taylorella asinigenitalis as Taylorella equigenitalis: implications for the epidemiology of CEM, J. Equine Vet. Sci. 20 (2000) 479.

[10] Sugimoto C., Isayama Y., Sakazaki R., Kuramochi S., Transfer of Haemophilus equigenitalis Taylor et al. 1978 to the genus Taylorella gen. nov. as Taylorella equigenitalis comb. nov., Curr. Microbiol. 9 (1983) 155-162.

[11] Taylor C.E.D., Rosenthal R.O., Brown D.F.J., Lapage S.P., Hill L.R., Legros R.M., The causative organism of contagious equine metritis 1977: proposal for a new species to be known as Haemophilus equigenitalis, Equine Vet. J. 10 (1978) 136-144.

[12] Widjojoatmodjo M.N., Fluit A.C., Verhoef J., Rapid identification of bacteria by PCR-SingleStrand Conformation Polymorphism, J. Clin. Microbiol. 32 (1994) 3002-3007. 